\title{
Coalgebras of Bounded Type
}

\author{
H. Peter Gumm and Tobias Schröder \\ Philipps-Universität Marburg \\ 35032 Marburg, Germany \\ \{gumm,tschroed\}@mathematik.uni-marburg.de
}

Received 16 May 2001

Using results of Trnková, we first show that subcoalgebras are always closed under finite intersections. Assuming that the type functor $F$ is bounded, we obtain a concrete representation of the terminal $F$-coalgebra. Several equivalent characterizations of boundedness are provided.

\section{Introduction}

Universal coalgebra as a theory of state based systems has seen a rapid development within recent years. For a long time the article (Rutten 2000) by J. Rutten has been the definite reference. Several of the results there have needed special conditions on the type functor, most prominently, preservation of weak pullbacks.

Recently, more and more results have been proved that freed the general theory from particular assumptions on the type functor. The existence of terminal coalgebras could be proven under the rather weak hypothesis that the type functor is bounded. It was only through some fruitful discussions with Jiři Adámek that led us to realize the connection to a class of functors which has been thoroughly investigated in the sixties by a group of mathematicians in Prague. As a result, it turns out in our main theorem that a functor $F$ is bounded if and only if there are sets $C$ and $M$ and a surjective natural transformation $\eta$ from the functor $C \times(-)^{M}$ to $F$.

To our surprise, we came across a result of Vera Trnková, another member of the Prague group, which shows that every nontrivial $\mathcal{S}$ et-endofunctor preserves nonempty pullbacks of monos. As a consequence, the collection of subcoalgebras of a fixed coalgebra is closed under finite intersections. Closure under arbitrary unions has been well known, so it turns out that the subcoalgebras of an arbitrary coalgebra form a topology.

This, and the main result of this paper show again, that the theory of coalgebras can not be obtained by straightforwardly dualizing the theory of universal algebra. One reason for this is that certain properties of the category $\mathcal{S} e t$ play an important role, and these properties are not valid in $\mathcal{S} e t^{o p}$, the dual of $\mathcal{S} e t$. 


\section{Preliminaries}

\subsection{Elementary notions}

A functor $F: \mathcal{C} \rightarrow \mathcal{D}$ between categories $\mathcal{C}$ and $\mathcal{D}$ associates with every object $A$ in $\mathcal{C}$ an object $F(A)$ in $\mathcal{D}$ and with every $\mathcal{C}$-morphism $f: A \rightarrow B$ a $\mathcal{D}$-morphism $F(f): F(A) \rightarrow$ $F(B)$ so that $F\left(i d_{A}\right)=i d_{F(A)}$ and $F(g \circ f)=F(g) \circ F(f)$ whenever $f: A \rightarrow B$ and $g: B \rightarrow C$.

A typical example is the powerset functor $\mathcal{P}: \mathcal{S}$ et $\rightarrow \mathcal{S}$ et which associates to every set $X$ its powerset $\mathcal{P}(X)=\{U \mid U \subseteq X\}$ and with every map $f: X \rightarrow Y$ the map $\mathcal{P}(f): \mathcal{P}(X) \rightarrow \mathcal{P}(Y)$ given by $\mathcal{P}(f)(U):=f[U]:=\{f(u) \mid u \in U\}$.

We shall almost exclusively restrict ourselves to set-endofunctors, that is to functors $F: \mathcal{S} e t \rightarrow \mathcal{S}$ et where $\mathcal{S}$ et denotes the category of sets. In the following we shall always assume that $F$ is nontrivial, in that $F(X) \neq \emptyset$ whenever $X \neq \emptyset .{ }^{\dagger}$

If $X \neq \emptyset$ and $f: X \rightarrow Y$ is an injective map, then $f$ has a left inverse, that is there exists a map $g: Y \rightarrow X$ with $g \circ f=i d_{X}$. Hence $F(g) \circ F(f)=i d_{F(X)}$, in particular, $F(f)$ is again injective. Similarly, if $f: X \rightarrow Y$ is surjective, then the axiom of choice yields a right inverse $g: Y \rightarrow X$ with $f \circ g=i d_{Y}$. Consequently, any set-endofunctor preserves surjectivity, and injectivity of maps with nonempty domain.

A natural transformation $\eta$ between set-endofunctors $G$ and $F$ consists of a map $\eta_{X}$ : $G(X) \rightarrow F(X)$ for every set $X$, so that for every map $f: X \rightarrow Y$ the obvious diagram commutes:

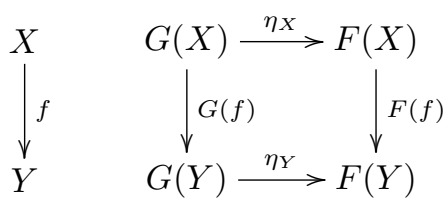

A natural transformation is called surjective, if $\eta_{X}$ is a surjective map whenever $X \neq \emptyset$.

\subsection{Coalgebras}

Any nontrivial functor $F: \mathcal{S} e t \rightarrow \mathcal{S}$ et will be called a type functor. A coalgebra $\mathcal{A}=$ $\left(A, \alpha_{A}\right)$ of type $F$ (also called $F$-coalgebra) consists of a set $A$ and a map $\alpha_{A}: A \rightarrow F(A)$. Here $A$ is called the carrier set and $\alpha_{A}$ is called the structure map.

By convention, we denote a coalgebra and its carrier set with the same letter and use different fonts for distinction. We also shall often drop the index to the structure map $\alpha$ when it is clear from the context.

2.2.1. Homomorphisms A map $\varphi: A \rightarrow B$ between coalgebras $\mathcal{A}=\left(A, \alpha_{A}\right)$ and $\mathcal{B}=$ $\left(B, \alpha_{B}\right)$ is a homomorphism, if $\alpha_{B} \circ \varphi=F(\varphi) \circ \alpha_{A}$, that is if the following diagram

\footnotetext{
$\dagger$ If $F X=\emptyset$ for some $X \neq \emptyset$, then $F Y=\emptyset$ for each $Y \neq \emptyset$. This is because there exists a map $Y \rightarrow X$ and hence a map $F Y \rightarrow F X=\emptyset$. In this case the category $\mathcal{S e t}_{F}$ consists of one single object, the empty $F$-coalgebra.
} 
commutes:

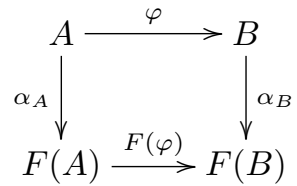

It is easy to check that the identity map $i d_{A}$ is always a homomorphism and that the composition $\psi \circ \varphi$ of two homomorphisms is a homomorphism again. Thus, $F$-coalgebras with their homomorphisms form a category which we shall denote by $\mathcal{S e t}_{F}$.

An isomorphism is a homomorphism with a both sided inverse. It is well known (Rutten 2000 ) that any bijective homomorphism is already an isomorphism. We call $\mathcal{A}$ and $\mathcal{B}$ isomorphic, and we write $\mathcal{A} \cong \mathcal{B}$, if there exists an isomorphism between $\mathcal{A}$ and $\mathcal{B}$.

2.2.2. Subcoalgebras If $\mathcal{A}$ is a coalgebra and $U \subseteq A$, we shall say that $U$ is a subcoalgebra of $\mathcal{A}$, provided that $U$ can be equipped with a coalgebra structure $\mathcal{U}=\left(U, \alpha_{U}\right)$ so that the set-theoretical inclusion map $\subseteq_{U}^{A}: U \hookrightarrow A$ is a homomorphism from $\mathcal{U}$ to $\mathcal{A}$.

The empty set $\emptyset$ is always a subcoalgebra, and if $\emptyset \neq U \subseteq A$, then $F\left(\subseteq_{U}^{A}\right)$ is injective, which entails that there can be at most one structure map $\alpha_{U}: U \rightarrow A$ making $\subseteq_{U}^{A}$ a homomorphism. Consequently, subcoalgebras are uniquely determined by their carrier sets.

2.2.3. Colimits and congruences The category $\mathcal{S}_{F}$ has arbitrary colimits, in fact, the forgetful functor from $\mathcal{S e t}_{F}$ to $\mathcal{S} e t$ creates colimits, see (Barr 1993; Barr 1994; Rutten 2000). Here we shall make use of the fact that every coalgebra $\mathcal{A}$ has a smallest homomorphic image. It can be obtained by forming the colimit (pushout) of all homomorphism with source $\mathcal{A}$. Alternatively, one may define a congruence to be the kernel of a homomorphism, then the smallest homomorphic image of $\mathcal{A}$ is obtained by factoring $\mathcal{A}$ through its largest congruence, see (Gumm and Schröder 2000).

Sums are particular examples of colimits. If $\left(\mathcal{A}_{i}\right)_{i \in I}$ is a family of coalgebras, then the sum $\Sigma_{i \in I} \mathcal{A}_{i}$ in $\mathcal{S e t}_{F}$ has as underlying set the disjoint union $\biguplus_{i \in I} A_{i}$ of the $A_{i}$ and as canonical homomorphisms $e_{i}: \mathcal{A}_{i} \rightarrow \Sigma_{i \in I} \mathcal{A}_{i}$ the set theoretical inclusion maps from $A_{i}$ into $\biguplus_{i \in I} A_{i}$

\subsection{Two examples}

In computer science, coalgebras model state based systems. The carrier set represents the state space and the structure map often encodes several components - some describing transitions to successor states and some representing various types of observations.

2.3.1. Automata For fixed sets $C$ and $M$ we consider the functor $F(X)=C \times X^{M}$. A map $f: X \rightarrow Y$ is assigned a map $F(f): C \times X^{M} \rightarrow C \times Y^{M}$ by means of $F(f)(c, \tau):=$ $(c, f \circ \tau)$.

An $F$-coalgebra $\mathcal{A}$ in this case consists of a set $A$ and a map $\alpha: A \rightarrow C \times A^{M}$. Obviously, $\alpha$ can equivalently be written as a combination of two components which we 
shall denote by $\gamma$ and $\delta$ :

$$
\begin{array}{ll}
\gamma: & A \rightarrow C \\
\delta & : A \times M \rightarrow A
\end{array}
$$

Thus, an $F$-coalgebra is just an automaton, where $\delta$ is the transition map, computing for every state $a \in A$ and every input $m \in M$ a new state $\delta(a, m)$, whereas $\gamma$ calculates an output $\gamma(a)$. Indeed, a coalgebra homomorphism $\varphi$ between two $F$-coalgebras $\mathcal{A}$ and $\mathcal{B}$ translates into the familiar notion of automata homomorphism:

$$
\begin{aligned}
\varphi\left(\gamma_{A}(a)\right) & =\gamma_{B}(\varphi(a)) \\
\varphi\left(\delta_{A}(a, m)\right) & =\delta_{B}(\varphi(a), m) .
\end{aligned}
$$

One easily checks that a subset $U \subseteq A$ is a subcoalgebra iff it is a subautomaton, that is if

$$
\forall u \in U . \forall m \in M . \delta(u, m) \in U .
$$

2.3.2. Nondeterministic automata In computer science, concurrency is often modelled by nondeterminism. In a nondeterministic automaton, the transition map is given the functionality

$$
\delta: A \times M \rightarrow \mathcal{P}(A)
$$

with $\mathcal{P}$ the powerset functor. Consequently, nondeterministic automata with input set $M$ and output set $C$ can be modelled as $F$-coalgebras where $F(X)=C \times \mathcal{P}(X)^{M}$.

\subsection{Terminal and cofree coalgebras}

Free algebras play a prominent role in universal algebra. In particular, the free algebra over the empty set of generators is initial in the category of algebras, in that it has precisely one homomorphism to each other algebra of the same type.

Dually, the terminal coalgebra, if it exists, incorporates all possible "behaviours" of coalgebras of the fixed type, see (Gumm 1999). For this reason, much effort has been devoted into showing the existence of terminal coalgebras for various functors.

Definition 2.1. An $F$-coalgebra $\mathcal{Q}$ is called terminal (or final), if for every $F$-coalgebra $\mathcal{A}$ there is a unique homomorphism $\varphi: \mathcal{A} \rightarrow \mathcal{Q}$. Without the "uniqueness" condition, $\mathcal{Q}$ is called weakly terminal.

From any weakly terminal coalgebra $\mathcal{W}$ the (unique) terminal coalgebra $\mathcal{Q}$ can be constructed by factoring $\mathcal{W}$ through its largest congruence.

2.4.1. The terminal automaton We shall now construct the terminal coalgebra for the functor $F(X)=C \times X^{M}$. By $M^{*}$ we denote the set of all finite words formed with elements from $M . \varepsilon$ is the empty word and for $m \in M$ and $w \in M^{*}$ we let $m \cdot w$ denote the word with first letter $m$ and rest $w$.

Now, the state set of the terminal automaton will be $Q:=C^{M^{*}}$, the set of all mappings 
from $M^{*}$ to $C$. Its transition structure is

$$
\begin{aligned}
\gamma_{Q}(\tau) & :=\tau(\varepsilon), \text { and } \\
\delta_{Q}(\tau, m)(w) & :=\tau(m \cdot w) .
\end{aligned}
$$

Given any automaton $\mathcal{A}$, one checks that the unique homomorphism to $\mathcal{Q}$ is given by $\varphi: A \rightarrow C^{M^{*}}$ where $\varphi(a): M^{*} \rightarrow C$ is recursively defined on $M^{*}$ by

$$
\begin{aligned}
\varphi(a)(\varepsilon) & =\gamma_{A}(a), \text { and } \\
\varphi(a)(m \cdot w) & =\varphi\left(\delta_{A}(a, m)\right)(w) .
\end{aligned}
$$

A good way to visualize an element $\tau \in Q$ may be as an infinite tree with nodes labeled by elements of $C$. Each node has $|M|$ sons and every word $w \in M^{*}$ denotes a path from the root to a particular node, or the "address" of that node. Thus $\gamma_{Q}(\tau)$ is the label of the root and $\delta_{Q}^{*}(\tau, w)$ is the node with address $w$ in the tree $\tau$.

2.4.2. Existence of terminal coalgebras In general, the existence of terminal coalgebras depends on the type functor $F$. In particular, it was observed by Lambek (see (Rutten 2000) for a proof) that the structure map $\alpha_{Q}: Q \rightarrow F(Q)$ on any terminal $F$-coalgebra must be a bijection. It follows that terminal coalgebras cannot exist in $\mathcal{S e t}_{\mathcal{p}}$. By the same token, there is no terminal nondeterministic automaton. We shall later consider a class of functors for which the existence of terminal coalgebras can be guaranteed.

2.4.3. Cofree coalgebras Just as initial algebras are special instances of free algebras, the terminal coalgebra, if it exists, is the same as the cofree coalgebra over a one-element set. The general definition is:

Definition 2.2. Let $X$ be a set, $\mathcal{S}_{X}$ an $F$-coalgebra and $\epsilon_{X}: S_{X} \rightarrow X$ a map. $\mathcal{S}_{X}$ with $\epsilon_{X}$ is called cofree over $X$, if for any $F$-coalgebra $\mathcal{A}$ and any map $\varphi: A \rightarrow X$ there is precisely one $F$-homomorphism $\tilde{\varphi}: \mathcal{A} \rightarrow \mathcal{S}_{X}$ with $\epsilon_{X} \circ \tilde{\varphi}=\varphi$.

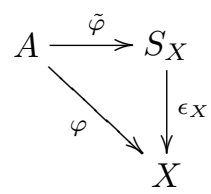

Cofree $F$-coalgebras over $X$ can be seen as terminal coalgebras for the functor $X \times F(-)$ with constant set $X$ :

If $\left(S_{X}, \alpha_{X}: S_{X} \rightarrow F\left(S_{X}\right)\right)$ with $\epsilon_{X}: S_{X} \rightarrow X$ is a cofree $F$-coalgebra over $X$, then $\left(S_{X},\left(\epsilon_{X}, \alpha_{X}\right): S_{X} \rightarrow X \times F\left(S_{X}\right)\right)$ is a terminal $X \times F(-)$-coalgebra.

On the other hand, if $\left(Q, \alpha_{Q}: Q \rightarrow X \times F(Q)\right)$ is a terminal $X \times F(-)$-coalgebra, we obtain a cofree $F$-coalgebra on $X$ by defining $S_{X}:=Q, \alpha_{X}:=\pi_{2} \circ \alpha_{Q}$ and $\epsilon_{X}:=\pi_{1} \circ \alpha_{Q}$ where $\pi_{1}: X \times F(Q) \rightarrow X$ and $\pi_{2}: X \times F(Q) \rightarrow F(Q)$ are the projections.

2.4.4. Cofree automata According to the above, the cofree automaton over $X$ with output set $C$ and input set $M$ can be constructed as the set of all infinite trees $\tau$ where each node has $|M|$ successors, and carries both a "label" from $C$ and a "color" from $X$. 
$\epsilon_{X}(\tau) \in X$ is the color and $\gamma(\tau) \in C$ is the label of the root. $\delta(\tau, m)$ is the subtree of $\tau$, whose root is the $m$-th son of $\tau$.

We leave it to the reader to check that that this defines the automaton with input $M$ and output $C$ which is cofree over $X$.

\subsection{The functor arising from a natural transformation}

Suppose that $\eta: G \rightarrow F$ is a natural transformation between set-endofunctors $G$ and $F$. Then $\eta$ gives rise to a functor $H_{\eta}: \mathcal{S e t}_{G} \rightarrow \mathcal{S e t}_{F}$ which is defined on objects as

$$
H_{\eta}\left(A, \alpha_{A}\right):=\left(A, \eta_{A} \circ \alpha_{A}\right)
$$

and which is the identity on morphisms.

Lemma 2.3. Let $\eta: G \rightarrow F$ be a natural transformation between nontrivial setendofunctors $G$ and $F$.

(i) Every subcoalgebra $U$ of $\mathcal{A} \in \mathcal{S e t}_{G}$ is an $F$-subcoalgebra of $H_{\eta}(\mathcal{A})$.

If additionally $\eta$ is surjective, then we have:

(ii) $H_{\eta}$ is surjective on objects, i.e. every coalgebra in $\mathcal{S e t}_{F}$ is of the form $H_{\eta}(\mathcal{A})$ for some $\mathcal{A}$ in $\operatorname{Set}_{G}$.

(iii)If $\mathcal{Q}$ is weakly terminal in $\mathcal{S e t}_{G}$ then $H_{\eta}(\mathcal{Q})$ is weakly terminal in $\mathcal{S e t}_{F}$.

(iv)If $\mathcal{S} e t_{G}$ has a terminal coalgebra $\mathcal{Q}$ then $\mathcal{S e t}_{F}$ has a terminal coalgebra $\mathcal{Q}^{\prime}$.

Proof. $(i)$ : If $\mathcal{U}=\left(U, \alpha_{U}\right)$ and $\mathcal{U} \leq \mathcal{A}$ then $\subseteq_{U}^{A}: \mathcal{U} \rightarrow \mathcal{A}$ is a $G$-homomorphism, so $H_{\eta}\left(\subseteq_{U}^{A}\right): H_{\eta}(\mathcal{U}) \rightarrow H_{\eta}(\mathcal{A})$ is an $F$-homomorphism. But $H_{\eta}$ is the identity on maps and on the carrier sets.

(ii) : Given $\mathcal{A}=(A, \alpha) \in \mathcal{S e t}_{F}$, the case $A=\emptyset$ is trivial. Otherwise, let $\left(\eta_{A}\right)^{-}: F(A) \rightarrow$ $G(A)$ be a right inverse of $\eta_{A}: G(A) \rightarrow F(A)$ and let $\mathcal{A}^{\prime}:=\left(A,\left(\eta_{A}\right)^{-} \circ \alpha\right)$. Then $H_{\eta}\left(\mathcal{A}^{\prime}\right)=\mathcal{A}$.

(iii) : Given an $F$-coalgebra $\mathcal{A}$, construct $\mathcal{A}^{\prime}$ as before, then there exists a $G$-homomorphism $\varphi: \mathcal{A}^{\prime} \rightarrow \mathcal{Q}$. Consequently, $\varphi=H_{\eta}(\varphi)$ is also an $F$-homomorphism from $\mathcal{A}=H_{\eta}\left(\mathcal{A}^{\prime}\right)$ to $H_{\eta}(\mathcal{Q})$.

(iv) : $\mathcal{Q}^{\prime}$ is the smallest factor of $\mathcal{H}_{\eta}(\mathcal{Q})$. It is obtained by factoring with the largest congruence relation.

\section{Intersections of subcoalgebras}

It is well known (for a proof see (Gumm 1999)) that the union of subcoalgebras of a fixed $F$-coalgebra $\mathcal{C}$ is again a subcoalgebra of $\mathcal{C}$. V. Trnková proves in (Trnková 1969) that every set-endofunctor preserves pullbacks given by nonempty intersections of two sets. From this one may infer that the intersection of two subcoalgebras of a given coalgebra $\mathcal{A}$ is again a subcoalgebra. We shall give a direct proof of the latter result:

Theorem 3.1. Let $F$ be an arbitrary $\mathcal{S}$ et-endofunctor and $\mathcal{A}$ an $F$-coalgebra. If $U$ and $V$ are subcoalgebras of $\mathcal{A}$ then so is $U \cap V$. 
Proof. $\emptyset$ is always a subcoalgebra, so we may assume $U \cap V \neq \emptyset$. We choose $w_{0} \in U \cap V$ and consider the maps $p: U \rightarrow U \cap V$ and $q: A \rightarrow V$ defined as

$$
p(u):=\left\{\begin{array}{ll}
u, & \text { if } u \in U \cap V ; \\
w_{0} & \text { otherwise, }
\end{array} \quad \text { and } q(a):= \begin{cases}a, & \text { if } a \in V ; \\
w_{0} & \text { otherwise. }\end{cases}\right.
$$

One observes the equalities

$$
q \circ \subseteq_{V}^{A}=i d_{V}
$$

and

$$
\subseteq_{U \cap V}^{V} \circ p=q \circ \subseteq_{U}^{A} .
$$

We now define a coalgebra structure $\beta$ on $U \cap V$ by

$$
\beta:=F(p) \circ \alpha_{U} \circ \subseteq_{U \cap V}^{U} .
$$

Here, and in all the following diagrams we denote set inclusions as well as their $F$-images by hooked arrows:

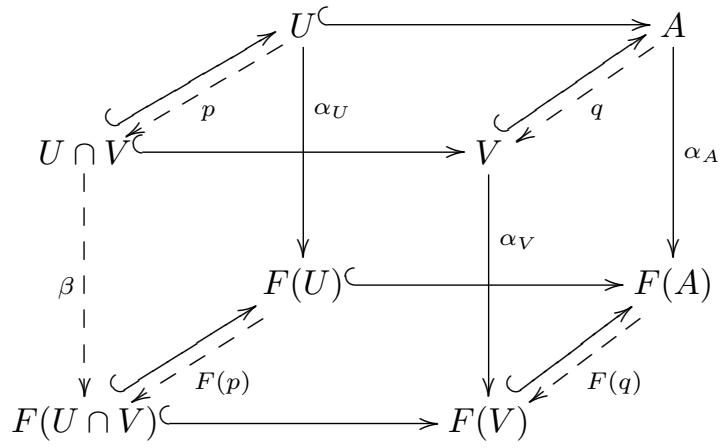

We now calculate:

$$
\begin{aligned}
F\left(\subseteq_{U \cap V}^{A}\right) \circ \beta & =F\left(\subseteq_{U \cap V}^{A}\right) \circ F(p) \circ \alpha_{U} \circ \subseteq_{U \cap V}^{U} \\
& =F\left(\subseteq_{V}^{A}\right) \circ F\left(\subseteq_{U \cap V}^{V} \circ F(p) \circ \alpha_{U} \circ \subseteq_{U \cap V}^{U}\right. \\
& =F\left(\subseteq_{V}^{A}\right) \circ F(q) \circ F\left(\subseteq_{U}^{A}\right) \circ \alpha_{U} \circ \subseteq_{U \cap V}^{U} \\
& =F\left(\subseteq_{V}^{A}\right) \circ F(q) \circ \alpha_{A} \circ \subseteq_{U}^{A} \circ \subseteq_{U \cap V}^{U} \\
& =F\left(\subseteq_{V}^{A}\right) \circ F(q) \circ \alpha_{A} \circ \subseteq_{U \cap V}^{A} \\
& =F\left(\subseteq_{V}^{A}\right) \circ F(q) \circ \alpha_{A} \circ \subseteq_{V}^{A} \circ \subseteq_{U \cap V}^{V} \\
& =F\left(\subseteq_{V}^{A}\right) \circ F(q) \circ F\left(\subseteq_{V}^{A}\right) \circ \alpha_{V} \circ \subseteq_{U \cap V}^{V} \\
& =F\left(\subseteq_{V}^{A}\right) \circ \alpha_{V} \circ \subseteq_{U \cap V}^{V} \\
& =\alpha_{A} \circ \subseteq_{V}^{A} \circ \subseteq_{U \cap V}^{V} \\
& =\alpha_{A} \circ \subseteq_{U}^{A} \cap
\end{aligned}
$$

This corollary cannot be improved in general to infinite intersections. In fact, in (Gumm $2001)$ it is shown that the filter functor $\mathcal{F}$ associating with every set $X$ the set $\mathcal{F}(X)$ 
of all filters on $X$ preserves finite intersection, but not infinite ones, so as a result, $\mathcal{F}$ subcoalgebras are not closed under arbitrary intersections.

As a consequence of the above, we can carry over to arbitrary type functors a result, which we had previous only been able to prove under additional assumptions in (Gumm and Schröder 2001).

Lemma 3.2. (i): If $\mathcal{U} \leq \Sigma_{i \in I} \mathcal{A}_{i}$ is a subcoalgebra of a sum of coalgebras, then $\mathcal{U} \cong$ $\Sigma_{i \in I}\left(\mathcal{U} \cap \mathcal{A}_{i}\right)$.

(ii): If $\mathcal{U}_{i} \leq \mathcal{A}_{i}$ for all $i \in I$, then $\Sigma_{i \in I} \mathcal{U}_{i} \leq \Sigma_{i \in I} \mathcal{A}_{i}$.

Proof. (i): Let $\mathcal{U} \leq \Sigma_{i \in I} \mathcal{A}_{i}$, then by the above, we have the subcoalgebras $U \cap A_{i}$ of $\mathcal{A}_{i}$. The universal property of the $\operatorname{sum} \Sigma_{i \in I}\left(\mathcal{U} \cap \mathcal{A}_{i}\right)$ gives us a unique homomorphism $\rho$ to $\mathcal{U}$ with $\rho \circ f_{i}=\subseteq_{U \cap A_{i}}^{U}$. As remarked in section 2.2.3, the forgetful functor creates colimits, thus the carrier set of $\Sigma_{i \in I}\left(\mathcal{U} \cap \mathcal{A}_{i}\right)$ is the disjoint union $\biguplus_{i \in I}\left(U \cap A_{i}\right)$ and the map $\rho$ is at the same time the unique set map from $\biguplus_{i \in I}\left(U \cap A_{i}\right)$ to $U$ satisfying $\rho \circ f_{i}=\subseteq_{U \cap A_{i}}^{U}$. In particular, $\rho$ is bijective, so by (Rutten 2000) it is an isomorphism.

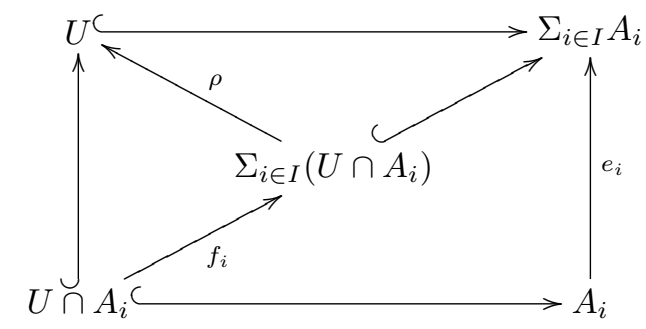

(ii): The universal map $\rho: \Sigma_{i \in I} \mathcal{U}_{i} \rightarrow \Sigma_{i \in I} \mathcal{A}_{i}$ is the same as the universal map between $\biguplus_{i \in I} U_{i}$ and $\biguplus_{i \in I} A_{i}$, i.e. the natural inclusion.

This lemma is particularly of interest when the closure of classes of coalgebras under class operators needs to be determined. For a class $\mathcal{K}$ of $F$-coalgebras let $\mathcal{S}(\mathcal{K})$, resp. $\Sigma(\mathcal{K})$, be the class of all subcoalgebras, resp. sums, of members of $\mathcal{K}$, then we have:

Corollary 3.3. For any class $\mathcal{K}$ of coalgebras

$$
\mathcal{S}(\Sigma(\mathcal{K}))=\Sigma(\mathcal{S}(\mathcal{K}))
$$

\section{Bounded, loosely bounded, and small functors}

The notion of a bounded functor has been defined in the context of coalgebras by J. Rutten. He calls a functor $F: \mathcal{S}$ et $\rightarrow \mathcal{S}$ et bounded, if there is a cardinal bound $\kappa$ for the size of one-generated coalgebras. In this case, the one-generated coalgebras form a set of generators, from which the existence of cofree coalgebras can be inferred. Ruttens definition, however, implicitly assumes that $F$ weakly preserves arbitrary intersections of infinitely many sets in order for the notion of one-generated subcoalgebras to make sense.

In (Gumm and Schröder 2000) we have therefore reformulated the definition of boundedness to make it apply to arbitrary $\mathcal{S}$ et-endofunctors. In the case where one-generated coalgebras exist, it obviously agrees with Rutten's definition: 
Definition 4.1. A functor $F: \mathcal{S} e t \rightarrow \mathcal{S}$ et is bounded by some set $M$, if for every $F$-coalgebra $\mathcal{A}$ and every $a \in A$ there exists a subcoalgebra $\mathcal{U}$ of $\mathcal{A}$ with $a \in U$ and $|U| \leq|M|$.

In (Gumm and Schröder 2000) we gave an elementary proof, showing that cofree $F$ coalgebras exist whenever $F$ is bounded. In this section we shall obtain a rather detailed characterization of bounded functors, theorem 4.7 below, from which an even more concrete construction of cofree coalgebras can be derived.

Lemma 4.2. For arbitrary sets $C$ and $M$, the functor $G(X):=C \times X^{M}$ is $\left|M^{*}\right|$-bounded.

Proof. From the description of $C \times X^{M}$-coalgebras as automata, one checks directly, that for every automaton $\mathcal{A}$ and for every $a \in A$ the smallest subautomaton $\langle a\rangle$ containing $a \in A$ is given by

$$
\left\{\delta^{*}(a, w) \mid w \in M^{*}\right\},
$$

where $\delta$ is the transition function of $\mathcal{A}$ (s. section 2.3.1) and $\delta^{*}: A \times M^{*} \rightarrow A$ is the extension of $\delta$ to $A \times M^{*}$ as defined in section 2.4.1. Consequently, $G(X)=C \times X^{M}$ is bounded by $M^{*}$.

As a direct consequence of lemma 2.3(ii) we also get:

Lemma 4.3. If $G$ is bounded, and $\eta: G \rightarrow F$ is a surjective natural transformation, then $F$ is bounded.

\subsection{Loosely bounded functors}

Definition 4.4. A set-endofunctor $F$ is called loosely bounded by some set $M$, if every nontrivial $F$-coalgebra contains a nontrivial subcoalgebra of size at most $|M|$.

Every bounded functor is loosely bounded. This follows from the fact that $F$ is bounded by $M$ if and only if every $F$-coalgebra $\mathcal{A}$ is the union of subcoalgebras of size at most $|M|$. Somewhat surprisingly, it will turn out as a consequence of theorem 4.7 that loosely bounded functors must already be bounded. Thus this definition will provide an easier criterion for checking boundedness.

\subsection{Small functors}

The notion of boundedness, which concerns any set-endofunctor $F$, refers to coalgebraic notions. It would be convenient, if it could be equivalently formulated without reference to coalgebras. We shall show that this is indeed possible. We use the notion of small functor that has been intensively studied by a group of mathematicians in Prague in the sixties, most prominently by J. Adámek, P. Goralčík, V. Koubek, and V. Trnková. Small functors are just the "accessible" Set-endofunctors (Adámek and Porst 2001).

Definition 4.5. Given a set $M$, a functor $F: \mathcal{S e t} \rightarrow \mathcal{S e t}$ is called $M$-small if $F(X)=$ $\bigcup\{F(\varphi)[F(M)] \mid \varphi: M \rightarrow X\}$ for every set $X \neq \emptyset$. 
Thus, the definition requires every element of $F(X)$ to be in the range of $F(\varphi)$ for an appropriate map $\varphi: M \rightarrow X$. A prototypical example of a bounded functor is given by the familiar $F(X)=C \times X^{M}$ :

Lemma 4.6. The functor $F(X)=C \times X^{M}$ is $M$-small.

Proof. Given an arbitrary $(c, \mu) \in C \times X^{M}$, then $\mu: M \rightarrow X$, so we simply choose $\left(c, i d_{M}\right) \in C \times M^{M}$ and obtain

$$
F(\mu)\left(c, i d_{M}\right)=\left(c, \mu \circ i d_{M}\right)=(c, \mu)
$$

\subsection{The characterization of bounded functors}

The following theorem brings together the notions of smallness and boundedness. As a consequence, we find that a functor $F$ is already bounded by $M$, if every $F$-coalgebra has a nonempty subcoalgebra of size at most $|M|$. The equivalence of $(i i i)$ and (iv) has been remarked in (Trnková 1969).

Theorem 4.7. Let $F: \mathcal{S} e t \rightarrow \mathcal{S}$ et be a nontrivial set-endofunctor. Then the following are equivalent:

(i) $F$ is bounded.

(ii) $F$ is loosely bounded.

(iii) $F$ is small.

(iv)There are sets $C$ and $M$ and a surjective natural transformation from $C \times(-)^{M}$ to $F$.

Proof. $(i) \rightarrow(i i)$ is trivial.

$($ ii $) \rightarrow($ iii $)$ : Given a set $X \neq \emptyset$ and $t \in F(X)$, we define a coalgebra structure on $X$ by the constant map $\alpha_{X}: X \rightarrow F(X)$ which takes every $x \in X$ to the element $t \in F(X)$. By assumption, $F$ is loosely bounded by some set $M$, so $\left(X, \alpha_{X}\right)$ has a nonempty subcoalgebra $U \leq X$ with $|U| \leq|M|$. We can therefore choose a surjective map $\psi: M \rightarrow U$. It follows that $F(\psi): F(M) \rightarrow F(U)$ is surjective too. Select an arbitrary $u \in U$, then we can find some $s \in F(M)$ with $F(\psi)(s)=\alpha_{U}(u)$. Now with $\varphi:=\subseteq_{U}^{X} \circ \psi$ we will have the map $\varphi: M \rightarrow A$, as required by the definition of smallness.

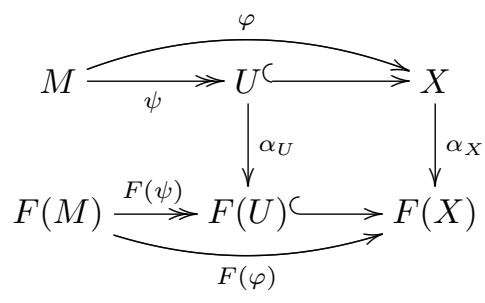


We can now calculate:

$$
\begin{aligned}
F(\varphi)(s) & =F\left(\subseteq_{U}^{X}\right) \circ F(\psi)(s) \\
& =F\left(\subseteq_{U}^{X}\right)\left(\alpha_{U}(u)\right) \\
& =\left(F\left(\subseteq_{U}^{X}\right) \circ \alpha_{U}\right)(u) \\
& =\left(\alpha_{X} \circ \subseteq_{U}^{X}\right)(u) \\
& =\alpha_{X}(u) \\
& =t .
\end{aligned}
$$

$($ iii $) \rightarrow($ iv $)$ : If $F$ is $M$-small, take $C:=F(M)$, and let $G(X):=C \times X^{M}$. Recall that for a map $f: X \rightarrow Y$ we have $G(f)(c, \mu)=(c, f \circ \mu)$. For any set $X$, we introduce the map $\eta_{X}: C \times X^{M} \rightarrow F(X)$ defined by

$$
\eta_{X}(c, \mu):=F(\mu)(c) .
$$

We check that $\eta: G \rightarrow F$ is a natural transformation: Let a map $f: X \rightarrow Y$ be given and any pair $(c, \mu) \in C \times X^{M}$, then

$$
\begin{aligned}
\left(F(f) \circ \eta_{X}\right)(c, \mu) & =F(f)(F(\mu)(c)) \\
& =(F(f) \circ F(\mu))(c) \\
& =F(f \circ \mu)(c) \\
& =\eta_{Y}(c, f \circ \mu) \\
& =\eta_{Y}(G(f)(c, \mu)) \\
& =\left(\eta_{Y} \circ G(f)\right)(c, \mu) .
\end{aligned}
$$

The fact that $F$ is $M$-small translates immediately into $\eta_{X}$ being surjective for every $X \neq \emptyset$.

$(i v) \rightarrow(i)$ combines lemmata 4.2 and 4.3 .

From the proof we observe that for the implications $(i) \rightarrow($ ii $) \rightarrow($ iii $) \rightarrow($ iv $)$ we can stay with the same bound $|M|$, whereas in the step $(i v) \rightarrow(i)$ we need to pass from $|M|$ to $\left|M^{*}\right|$, as a consequence of lemma 4.2. Consequently we obtain as a corollary:

Corollary 4.8. If $M$ is an infinite set, then the following are equivalent:

(i) $F$ is bounded by $M$.

(ii) $F$ is loosely bounded by $M$.

(iii) $F$ is $M$-small.

(iv)There is a set $C$ and a surjective natural transformation from $C \times(-)^{M}$ to $F$.

As a consequence of theorem 4.7, coalgebras with bounded type functors can be naturally derived from automata. From this, we easily infer the closure of bounded functors under sums, products, and compositions:

Corollary 4.9. If $F$ and $F^{\prime}$ are bounded, then so are $F+F^{\prime}, F \times F^{\prime}$, and $F \circ F^{\prime}$.

Proof. Sum, product and composition of $F=C \times(-)^{M}$ with $F^{\prime}=C^{\prime} \times(-)^{M^{\prime}}$ are obviously bounded. It remains to verify that from surjective natural transformations 
$\eta: G \rightarrow F$ and $\eta^{\prime}: G^{\prime} \rightarrow F^{\prime}$ one obtains surjective natural transformations $\eta+\eta^{\prime}$ : $G+G^{\prime} \rightarrow F+F^{\prime}, \eta \times \eta^{\prime}: G \times G^{\prime} \rightarrow F \times F^{\prime}$, and $\eta^{\prime \prime}: F \circ F^{\prime} \rightarrow G \circ G^{\prime}$, where the latter is defined on any set $X$ as

$$
\eta_{X}^{\prime \prime}:=G\left(\eta_{X}^{\prime}\right) \circ \eta_{F^{\prime}(X)} .
$$

\section{The existence of terminal and cofree coalgebras}

It is well known that terminal coalgebras exist for bounded type functors. Boundedness of $F$ guarantees the existence of a set of generators, so an application of the special adjoint functor theorem (see (Borceux 1994)) would guarantee the existence of the terminal coalgebra. In (Gumm and Schröder 2000) we have given a direct construction.

As a consequence of our main theorem 4.7, however, we even know how the terminal coalgebra arises from the terminal automaton. If $\mathcal{Q}$ is the terminal automaton, as described in subsection 2.4.1, then the terminal $F$-coalgebra is the smallest factor of $H_{\eta}(\mathcal{Q})$, where $\eta$ is the surjective natural transformation from $G(-)=C \times(-)^{M}$ to $F$.

Cofree coalgebras can be constructed in a similar manner. We have already remarked how the cofree $F$-coalgebra over $X$ arises from the terminal coalgebra of type $X \times F(-)$. If $F$ is bounded, then so is $X \times F(-)$, for a surjective natural transformation $\eta: C \times(-)^{M} \rightarrow$ $F(-)$ can be extended to a surjective natural transformation

$$
i d_{X} \times \eta: X \times C \times(-)^{M} \rightarrow X \times F(-) .
$$

\subsection{An application: Terminal $\mathcal{P}_{\omega}$-coalgebras}

To see a concrete example, consider the finite powerset functor $\mathcal{P}_{\omega}$ which associates with every set $X$ the set of all finite subsets of $X$. Given a map $f: X \rightarrow Y$ let $\mathcal{P}_{\omega}(f)(U):=f[U]$ for every finite subset $U \subseteq X$. With $C:=\mathcal{P}_{\omega}(\omega)$ and $M:=\omega$, we construct a natural transformation $\eta: C \times(-)^{M} \rightarrow \mathcal{P}_{\omega}(-)$. by defining for every set $X$ :

$$
\eta_{X}(U, \mu):=\mu[U] .
$$

One easily checks that $\eta$ is natural and surjective, hence $\mathcal{P}_{\omega}$ is bounded by $\omega$.

By lemma 2.3 , the terminal $\mathcal{P}_{\omega}$-coalgebra is the smallest quotient of $H_{\eta}(\mathcal{Q})$ where $\mathcal{Q}$ is the automaton with state set $\mathcal{P}_{\omega}(\omega)^{\omega^{*}}$.

$Q$ can be visualized as the set of all countably branching infinite trees $\tau$, where each node is labeled by some finite subset of $\omega$. For $n \in \omega$, let $\tau_{n}:=\lambda w \cdot \tau(n \cdot w)$ be the $n$-th son of $\tau$.

Thus, $\mathcal{Q}=\left(\mathcal{P}_{\omega}(\omega)^{\omega^{*}}, \alpha_{Q}\right)$, where $\alpha_{Q}(\tau)=\left(\tau(\varepsilon), \lambda m . \delta_{Q}(\tau, m)\right)=\left(\tau(\varepsilon), \lambda m . \tau_{m}\right)$. Now $\mathcal{H}_{\eta}(\mathcal{Q})=\left(Q, \eta_{Q} \circ \alpha_{Q}\right)$ where

$$
\left(\eta_{Q} \circ \alpha_{Q}\right)(\tau)=\left\{\tau_{m} \mid m \in \tau(\varepsilon)\right\} .
$$




\subsection{The terminal image finite transition system}

$\mathcal{P}_{\omega}$-coalgebras are also known as image finite transition systems. A structure map $\alpha$ : $A \rightarrow \mathcal{P}_{\omega}(A)$ corresponds uniquely to a binary relation $R_{\alpha} \subseteq A \times A$ where for every $a \in A$, the set $\{b \in A \mid a R b\}$ is finite. Such a binary relation can be interpreted as a transition relation, in which case it is customary write

$$
a \rightarrow b
$$

instead of $a R b$. For the above coalgebra $\mathcal{H}_{\eta}(\mathcal{Q})$ this reads as

$$
\tau \rightarrow \sigma \Longleftrightarrow \exists n \in \tau(\varepsilon) . \sigma=\tau_{n} .
$$

This $\mathcal{P}_{\omega}$-coalgebra is weakly terminal. In order to obtain the terminal coalgebra, one has to factor by the largest congruence relation. It is well known that the functor $\mathcal{P}_{\omega}$ weakly preserves pullbacks, so the largest congruence relation is the same as the largest bisimulation (see (Rutten 2000) for these notions). In our context, this is the largest relation $\sim$ on $Q$, satisfying:

$\tau \sim \sigma$ implies

(i) $\forall m \in \tau(\varepsilon) . \exists n \in \sigma(\varepsilon) \cdot \tau_{m} \sim \sigma_{n}$, and

(ii) $\forall n \in \sigma(\varepsilon) . \exists m \in \sigma(\varepsilon) . \tau_{n} \sim \sigma_{m}$.

Thereby we have obtained an easy characterization of the terminal $\mathcal{P}_{\omega}(-)$ coalgebra. It may be instructive to compare this description with those given by M. Barr (Barr 1993; Barr 1994) or J. Worrel (Worrell 1999).

\section{Conclusion}

For arbitrary coalgebras we have observed that subcoalgebras are closed under finite intersections, hence the formation of sums and subcoalgebras commute.

For bounded functors $F$, we have given a rather detailed description of $F$-coalgebras using a surjective natural transformation from a particular automaton. This description appears to be useful for characterizing cofree $F$-coalgebras.

While the paper was under the reviewing process, J. Adámek and H. Porst have given in (Adámek and Porst 2001) an example for an unbounded functor that does have cofree coalgebras over every color set $X$. In this paper there is also a proof for the fact that small functors are just the accessible $\mathcal{S}$ et-endofunctors.

\section{References}

J. Adámek and H. Porst, From varieties of algebras to covarieties of coalgebras, Coalgebraic Methods in Computer Science (CMCS'01) (A. Corradini, M. Lenisa, and U. Montanari, eds.), Electronic Notes in Theoretical Computer Science, no. 44.1, Elsevier, 2001, pp. 27-46.

M. Barr, Terminal coalgebras in well-founded set theory, Theoretical Computer Science (1993), no. 114(2), 299-315.

M. Barr, Additions and corrections to 'terminal coalgebras in well-founded set theory', Theoretical Computer Science (1994), no. 124(1), 189-192. 
F. Borceux, Handbook of categorical algebra 1: basic category theory, Cambridge University Press, 1994.

H.P. Gumm and T. Schröder, Covarieties and complete covarieties, Theoretical Computer Science (2001), no. 260 (1-2), 71-86.

H.P. Gumm and T. Schröder, Products of coalgebras, Algebra Universalis (2001), no. 46, 163-185.

H.P. Gumm, Functors for coalgebras, Algebra Universalis (2001), no. 45 (2-3), 135-147.

H.P. Gumm, Elements of the general theory of coalgebras, LUATCS 99, Rand Afrikaans University, Johannesburg, South Africa, 1999.

J.J.M.M. Rutten, Universal coalgebra: a theory of systems, Theoretical Computer Science (2000), no. $249,3-80$.

V. Trnková, Some properties of set functors, Comm. Math. Univ. Carolinae (1969), no. 10,2, 323-352.

J. Worrell, Terminal sequences for accessible endofunctors, Coalgebraic Methods in Computer Science (B. Jacobs et al, ed.), Electronic Notes in Theoretical Computer Science, vol. 19, Elsevier Science, 1999, pp. 39-53. 